\title{
The relationship between the malignancy grade of lung adenocarcinoma with micropapillary pattern and the findings of positron emission tomography
}

\author{
Norifumi Tsubokawa' ${ }^{1}$ Takahiro Mimae ${ }^{2}$, Yasuhiro Tsutani ${ }^{2}$, Takeshi Mimura ${ }^{2}$, Yoshihiro Miyata ${ }^{2}$, Morihito Okada ${ }^{2 *}$ \\ From World Society of Cardiothoracic Surgeons 25th Anniversary Congress, Edinburgh \\ Edinburgh, UK. 19-22 September 2015
}

\section{Background/Introduction}

The survival rates are not always high after the complete resection even if early stage lung adenocarcinoma. Micropapillary pattern (MPP) was one of prognostic factors in such cancer.

\section{Aims/Objectives}

This study aimed to investigate whether preoperative maximum standard uptake value (SUVmax) on positron emission tomography/computed tomography (PET/CT) could indicate early stage lung adenocarcinoma with MPP.

\section{Method}

Total 347 consecutive patients with clinical stage IA lung adenocarcinoma that were treated by complete resection were retrospectively examined. We defined MPP- positive as accounting for $5 \%$ or more of the entire tumor.

\section{Results}

Forty eight (14\%) and 299 (86\%) patients were MPPpositive and negative, respectively. There were no significant differences between both groups in age $(\mathrm{P}=0.369)$, gender $(P=0.059)$, or tumour size $(P=0.437)$. However, SUVmax on PET/CT were significantly higher in MPPpositive, than negative group $(3.02 \pm 2.34$ vs. $2.19 \pm 2.45$, $\mathrm{P}=0.029$, respectively). In addition, lymphatic and vascular invasion as well as lymph node metastasis were more frequent in the MPP-positive, than negative group $(\mathrm{P}=0.003, \mathrm{P}=0.029$, and $\mathrm{P}=0.002$, respectively). Fiveyear recurrence free survival (RFS) rates were significantly lower in the MPP-positive, than negative group (69.7\% vs. $89.3 \%, \mathrm{P}<0.001)$. Multivariate analysis for RFS showed that MPP, lymphatic and vascular invasion were independent poor prognostic factors $(\mathrm{P}=0.048, \mathrm{P}=0.003$, $\mathrm{P}=0.002$, respectively).

\section{Discussion/Conclusion}

The presence $(\leq 5 \%)$ of MPP in early stage lung adenocarcinoma should be considered a distinct subtype with a high risk of recurrence and a poor prognosis. In addition, preoperative PET/CT was useful for predicting whether tumours harboured MPP or not.

\section{Authors' details}

'Department of Respiratory Surgery, National Hospital Organization Kure Medical Centre and Chugoku Cancer Centre, Kure, Hiroshima, 737-002, Japan. ${ }^{2}$ Department of Surgical Oncology, Hiroshima University, Hiroshima, 734-8551, Japan

Published: 16 December 2015

\section{doi:10.1186/1749-8090-10-S1-A152}

Cite this article as: Tsubokawa et al:: The relationship between the malignancy grade of lung adenocarcinoma with micropapillary pattern and the findings of positron emission tomography. Journal of Cardiothoracic Surgery 2015 10(Suppl 1):A152. 